\title{
Medically inoperable peripheral lung cancer treated with stereotactic body radiation therapy
}

\author{
K. D. Kelley ${ }^{1}$, D. L. Benninghoff ${ }^{2}$, J. S. Stein ${ }^{3}$, J. Z. Li ${ }^{2}$, R. T. Byrnes ${ }^{2}$, L. Potters ${ }^{1}$, J. P. S. Knisely ${ }^{1}$ and H. D. Zinkin ${ }^{2,4^{*}}$
}

\begin{abstract}
Background: Lung cancer is the most frequent cause of cancer-related death in North America. There is wide variation between patients who are medically inoperable and those managed surgically. The use of stereotactic body radiotherapy (SBRT) has narrowed the gap in survival rates between operative and non-operative management for those with early stage disease. This retrospective study reports outcomes for the treatment of peripheral non-small cell lung carcinoma (NSCLC) with SBRT from a single community practice.
\end{abstract}

Methods: Sixty-seven consecutive patients (pts) with inoperable, untreated peripheral lung tumors were treated from 2010 through 2012 and included in this study. Stereotactic targeting was facilitated by either spine or lung-based image guidance, either with or without fiducial marker tracking with a frameless robotic radiosurgery system. Peripheral tumors received a median biological effective dose (BED) of $105.6 \mathrm{~Gy}_{10}$ or in terms of a median physical dose, $48 \mathrm{~Gy}$ delivered over 4 daily fractions. Survival was measured using the Kaplan-Meier method to determine rates of local control, progression of disease and overall survival. The Cox proportional hazards regression model was used to study the effects of tumor size, stage, histology, patient age, tumor location (lobe), tracking method, and BED on the survival distributions.

Results: The median follow-up for this cohort was 24.5 months (range: 2.4-50.3) with an overall (OS) 3-year survival of $62.4 \%$ (95 \% Cl: 74.3-47.3). The median progression-free survival was 28.5 months (95\% Cl: 15.8 months to not reached). Local control (LC), defined as a lack of FDG uptake on PET/CT or the absence of tumor growth was achieved in 60 patients (90.9\%) at the time of first follow-up (median 3 months, range: 1-6). Local control at one year for the entire cohort was $81.8 \%(95 \% \mathrm{Cl}, 67.3-90.3)$. The one-year OS probability among those who achieved local control at first follow-up was $86.2 \%(95 \% \mathrm{Cl}, 74.3-92.9)$ but no patients who did not achieve LC at first follow-up survived one year. Of the 60 pts that achieved initial LC, 16 have died. The rates of local control, progression-free survival and overall survival were not statistically different for patients treated using a fiducial target tracking system versus non-invasive guidance. ( $p=0.44, p=0.97$ and $p=0.66$, respectively). No National Cancer Institute (NCl) Common Terminology Criteria for Adverse Events (CTCAE-4) grade 3 or greater toxicity was observed.

Conclusion: SBRT is an effective treatment for medically inoperable NSCLC patients with peripherally located tumors. This therapy appears to be well tolerated with low toxicity, and patient outcomes when using non-invasive tumor tracking systems are not inferior to traditional fiducial-based techniques.

\footnotetext{
*Correspondence: hzinkin@nshs.edu

${ }^{2}$ The Department of Radiation Medicine, North Shore-LIJ Health System, Huntington, NY, USA

${ }^{4} 989$ W Jericho Turnpike, Smithtown, NY 11787, USA

Full list of author information is available at the end of the article
} 


\section{Background}

Until the advent of stereotactic body radiation therapy (SBRT), patients with early and intermediate stage lung cancer who were medically inoperable had limited treatment options [1]. Traditional protracted radiotherapy yielded relatively poor outcomes for this patient population historically with a 5-year overall survival of only 13-39\% [2]. Sophisticated treatment planning and image guidance now permits focal high-dose per fraction radiation to be delivered safely $[3,4]$. This approach of dose escalation delivered to a defined target while preserving adjacent healthy tissues with strict geometric avoidance of organs at risk (OARs) is what constitutes SBRT. Clinical trials have shown an improvement of the therapeutic ratio compared to results from conventional radiotherapy with enhanced control rates and low toxicity to OARs [1,5-11]. This approach has in turn proven very effective in controlling tumors in medically inoperable patients and is now considered a standard of care for this patient population [1].

The purpose of this study was to analyze the outcomes of patients treated with SBRT for early and intermediate stage NSCLC within a community-based setting, in order to outline potential prognostic factors and assess variations in survival and local control rates when using different methods of image guidance. Moreover, data analyzed from a community-based practice may better estimate non-academic centers' outcomes and be more representative of likely outcomes outside tertiary centers. Additionally, lower dose per fraction regimens, similar to those used in this study and known to carry less risk of inducing acute and long-term toxicities, are hypothesized to be more routinely used in a community setting, as opposed to higher dose-escalation strategies reported by many early multi-institutional series done at large academic centers $[1,12]$.

Here we outline as our primary endpoint, all-cause mortality reported as overall survival of the entire cohort measured from the time of therapy completion. Other endpoints include disease-free survival, local control, and correlations between local control and overall survival including tumor size, histology, tumor location, and treatment technique.

\section{Methods}

Study approval was obtained from the North Shore-LIJ Health System (NSLIJHS), Monter Cancer Center Scientific Advisory Committee. A retrospective chart review protocol was further reviewed and approved by the NSLIJHS Institutional Review Board (IRB). Thereafter, a database of all patients diagnosed with lung cancer and treated with radiosurgery at North Shore Radiation Therapy from 2010 to 2012 was created to allow for at least a minimum of 2 years of follow-up. The following variables were collected during the analysis: date of treatment, patient age, tumor histology, tumor size, technique of treatment, tumor location, length of follow-up, toxicity, tumor control, and survival. IRB policies were followed regarding data deidentification.

\section{Radiotherapy technique and specifications}

Due to the presence of a collaborative thoracic oncology program with prospective review, each patient was evaluated by a multidisciplinary team including a thoracic surgeon, interventional radiologist, medical and radiation oncologists. Patients were deemed medically inoperable based on objective criteria including a FEV1 $<1.5 \mathrm{~L}$, preoperative FEV1 $<40 \%$ predicted value or DLCO $<40 \%$ of predicted value and at the final discretion of the surgeon. Many patients included in this study had fiducial markers placed by interventional radiology at the time of tumor biopsy unless they were deemed too high risk for this procedure. All patients were then simulated on a GE Lightspeed 64 slice 4D CT simulator and treated at North Shore Radiation Therapy/Cyberknife of Long Island. Target structures were delineated on pulmonary $\mathrm{CT}$ windows with the aid of PET fusion. Avidity (a sustained uptake value (SUV) of greater than 2) was included as part of gross tumor volume (GTV). For patients without fiducial markers or tumors not large enough to be seen and tracked using orthogonal radiographs, a 4D CT of the chest was used to account for tumor motion and create an internal target volume (ITV). The ITV was expanded 3-5 $\mathrm{mm}$ to account for set up error and arrive at the planning target volume (PTV). The total dose was prescribed to the $80 \%$ isodose volume. The median biological BED used in this study was 105.6 Gy (range, 180-85.5 Gy (10 $_{10}$ ). Organs at risk and their respective tolerance doses used during treatment planning are shown in Table 1.

Three image guidance systems onboard the Cyberknife platform were used: the XSight Spine Tracking System, which relies on bony anatomy of the spine to locate and

Table 1 Organ tolerance dose limits

\begin{tabular}{lcl}
\hline Organ total dose volume & & \\
\hline Spinal cord & 22 Gy Maximum & To any point \\
Esophagus & 27 Gy maximum & To any point \\
Brachial plexus & 24 Gy maximum & To any point \\
Aorta & 45 Gy maximum & To any point \\
Heart & 30 Gy maximum & To any point \\
Trachea and bronchus & 30 Gy maximum & To any point \\
Chest wall/ ribs & 30 Gy maximum ${ }^{\text {a }}$ & To any point \\
Both lungs & 20 Gy & $<10 \%$ of organ volume \\
Both Lungs & 15 Gy & $<35 \%$ of organ volume \\
Skin & 24 Gy maximum ${ }^{\text {a }}$ & To any point \\
\hline
\end{tabular}

${ }^{a}$ considered for superficial tumors $<2 \mathrm{~cm}$ from chest wall 
track tumors; the Synchrony Respiratory Motion Tracking System, which continuously synchronizes beam delivery with the motion of the target resulting from respiration by using external fiducial markers as a surrogate to track tumor motion; and the XSight Lung Tracking System, which tracks the soft tissue (tumor) target with respiration without the need for fiducial markers [13].

\section{Statistical analysis}

Statistical objectives of this study included the estimation of median time until local progression, disease-free survival, all-cause mortality and acute toxicity. Survival was measured using the Kaplan-Meier method to characterize the progression of disease, local control and survival distributions. Median time to the event was estimated along with a $95 \%$ confidence interval $(\mathrm{CI})$. The Cox proportional hazards regression model was used to study the effects of multiple predictor variables (e.g. tumor size, stage, histology, patient age, tumor location (lobe), tracking method and biologic effective dose (BED)) on these three time-until-event outcomes. Descriptive statistics (proportions for categorical variables and means and standard deviations for continuous variables) were also calculated. Survival time was measured in months. Time until death was calculated from date of treatment completion to either date of event, or date of censoring (date of last follow-up), except for local failure at first follow-up scan post-treatment. In this case, time until death was measured from the date of first follow-up scan and not the date of treatment completion. For disease-free survival, failure was defined as any of the following: local failure, regional failure, distant failure, or locoregional failure. In cases where the event of death was not observed, the number of months until last follow-up was used and the subject's vital status was classified as censored. The effects of categorical demographic, clinical, pathologic, and treatment variables were assessed using the log-rank test. Univariate Cox regression was used for continuous variables. A result was considered statistically significant if the $\mathrm{p}$ value was $<0.05$. To adjust for tied failure times, Efron's method was used [14].

\section{End points and follow-up}

Approximately three months after completing treatment, a follow-up radiologic examination was performed to determine the initial response to treatment. Two patients were followed by a non-contrast $\mathrm{CT}$ and the remaining with PET-CT scans. After the initial follow-up period, a chest CT or PET-CT scan was done to evaluate both tumor size and metabolism every 3 to 6 months for two years post treatment --after which follow-up was done on an annual basis.

The definition of local control used in the current analysis was based on that used in RTOG 0236 [15]. Local failure was defined as meeting any one of the following criteria: (1) local tumor enlargement greater than $20 \%$ of the gross tumor volume compared to the treatment planning CT-scan, (2) evidence of increasing metabolism using PET imaging, or (3) development of a new lesion in the involved lobe [15]. Regional failure was defined as a recurrence within a different ipsilateral lobe or any regional lymph node station including the bilateral hilar, mediastinal, scalene, or supraclavicular nodal stations as defined in the American Joint Committee on Cancer (AJCC) Cancer Staging Manual, $7^{\text {th }}$ edition [16]. Distant spread was defined as either radiographic evidence of a malignant pleural or pericardial effusion, pleural-based nodules, contralateral lung nodules, distant solid organ, CNS or osseous involvement.

\section{Results}

A total of 67 medically inoperable patients with early and intermediate stage lung cancer were treated with stereotactic body radiotherapy (SBRT) between February 5, 2010 and November 4, 2013 (Table 2). Each patient had a single peripheral tumor defined as at least $2 \mathrm{~cm}$ from the trachea, carina and mainstem bronchi. One patient had limited follow-up, and was therefore included only in the overall survival analysis. Of the remaining 66 patients: 43 had local control, 11 failed locally, 5 regionally, 2 distantly and 5 locoregionally. Twenty-two of the 67 subjects eventually died. Twenty-three of 66 subjects had disease progression, and 6 had failed locally at the time of initial post-treatment evaluation. The mean follow-up was 24.5 months (range: 2.4-50.3 months). Among subjects who remained alive or were censored, the mean follow-up time was 28.6 months (range: 2.450.3 months).

\section{Overall survival based on local control status at first follow-up}

All patients were imaged 1-6 months (median 3 months) after treatment. Due to the variability in how long after treatment a first follow-up scan was obtained, a sensitivity analysis was performed whereby the actual follow-up time from treatment to time of scan was adjusted for in a Cox regression model with local control status at time of first scan as the predictor of interest. The results from this analysis (data not shown) were similar to the Kaplan Meier estimate thus, only the Kaplan Meier results are reported as representative of local control status.

The achievement of local control at the time of initial follow-up was significantly associated with improved overall survival. Local control data were available for 66 patients. Among the 60 patients with local control determined at first follow-up, $16 / 60$ died, whereas all 6 of the subjects who failed locally at their first follow-up scan succumbed to their disease. The estimated 1 year survival 
Table 2 Patient characteristics $(N=67)$

\begin{tabular}{|c|c|c|}
\hline \multicolumn{2}{|c|}{ Age, years, median (range) } & \multirow{2}{*}{$\begin{array}{l}79(60-92) \\
n=24(36 \%)\end{array}$} \\
\hline Sex & Male & \\
\hline & Female & $n=43(64 \%)$ \\
\hline \multirow[t]{2}{*}{ Stage } & IA & $n=52(78 \%)$ \\
\hline & $|\mathrm{B}-|||$ & $n=15(22 \%)$ \\
\hline \multirow[t]{4}{*}{ Location } & Left Lower lobe & $n=16(24 \%)$ \\
\hline & Left Upper Lobe & $n=16(24 \%)$ \\
\hline & Right Lower Lobe & $n=12(18 \%)$ \\
\hline & Right Upper Lobe & $n=23(34 \%)$ \\
\hline \multirow[t]{5}{*}{ Tumor volume } & $<2.5 \mathrm{cc}$ & $n=17(25 \%)$ \\
\hline & $5-2.5 \mathrm{cc}$ & $n=19(29 \%)$ \\
\hline & $5-10 c c$ & $n=7(11 \%)$ \\
\hline & $10-20 c c$ & $n=9(13 \%)$ \\
\hline & $>20 \mathrm{cc}$ & $n=15(22 \%)$ \\
\hline \multirow[t]{2}{*}{$B E D_{10}^{a}$} & Mean & 107.8 \\
\hline & Median (Range) & $105.6(180-85.5)$ \\
\hline \multirow[t]{3}{*}{ Tracking } & XSight-spine & $n=30(45 \%)$ \\
\hline & XSight-lung & $n=3(5 \%)$ \\
\hline & XSight-spine + Fiducials & $n=34(50 \%)$ \\
\hline \multirow[t]{6}{*}{ Histology } & Adenocarcinoma & $n=30(44 \%)$ \\
\hline & Squamous Cell Carcinoma & $n=14(21 \%)$ \\
\hline & Non-small cell lung carcinoma-NOS ${ }^{b}$ & $n=14(21 \%)$ \\
\hline & Carcinoid & $n=3(4 \%)$ \\
\hline & Sarcomatoid mesothelioma & $n=1(2 \%)$ \\
\hline & Non-diagnostic biopsy & $n=6(8 \%)$ \\
\hline
\end{tabular}

${ }^{a}$ Biologic Effective Dose, $\mathrm{Gy}_{10}$ or $\alpha / \beta$ ratio $=10$

${ }^{\mathrm{b}}$ Not otherwise specified

probability among patients achieving local control was $86.2 \%$ (95 \% CI, 74.3-92.9), whereas the one year survival probability for patients with local failure at first follow-up scan was $0.0 \%$ (95 \% CI, not estimable) (Fig. 1).

Local control, disease-free and overall survival rates

At 12 months, $81.8 \%$ (95\% CI, 67.3-90.3) of patients maintained local control out of the total cohort. However, by 24 months only $60.6 \%$ (95\% CI, 41.5- 75.2) of patients had local control although the median time to local progression of disease was never reached (Fig. 2). The overall disease-free survival at 3 years was $37.5 \%$ (95 \% CI: 17.7 to 57.4). Median disease-free survival was estimated to be 28.5 months (95 \% CI: 15.8 months to not reached) (Fig. 3a). The overall survival at 3 years was $62.4 \%$ (95\% CI: 74.3-47.3 \%). The median overall survival time was not reached during the designated follow-up period (Fig. 3b).

\section{Comparative analysis of survival outcomes}

A comparison of survival outcomes stratified by various patient and treatment-specific factors was not found to be statistically significant (using the log-rank test and $p<0.05)$. For example, analysis of time until disease progression with respect to gender $(p=0.8)$, stage $(p=0.16)$, pathology $(p=0.1)$, or tumor location/lobe involved $(p=0.15)$ did not reveal any significant differences (Additional file 1: Figure S1). Similarly, non-significant results were found in Cox regression analyses for $\mathrm{BED}_{10}$ $(p=0.43)$, age $(p=0.15)$, and tumor size $(p=0.66)$ with respect to disease-free survival (data not shown). Kaplan-Meier analysis for time until death was not found to be statistically significant among the following subgroups by univariate analyses: gender $(p=0.13)$, stage $(p=0.37)$, pathology $(p=0.77)$, and tumor location/lobe $(p=0.81)$ (Additional file 2: Figure S2). Results from a univariate Cox regression analysis did not provide statistical evidence to suggest a difference in overall survival according to $\operatorname{BED}_{10}(p=0.08)$, age $(p=0.74)$, or tumor size $(p=0.29)$ (data not shown).

\section{Survival outcomes based on tracking method}

A comparison of overall survival in patients stratified by motion tracking and targeting system used during treatment was also done. Either non-invasive image guidance (XSight-spine/lung) alone or XSight-spine followed by matching to surgically implanted-intratumoral fiducials was used to localize and track tumors during each fraction of treatment. In the latter technique, tumors were initially aligned for treatment using the osseous spinal anatomy of each patient as a surrogate (XSight-spine) and then further adjusted through matching to radioopaque fiducials within the tumor. In the event that tumor size was sufficient to visualize on orthogonal radiographs, XSight-lung localization was utilized (3 patients out of 67) after an initial matching to a portion of bony spine adjacent to the target. Survival was not found to be statistically different in patients treated using XSight-spine/lung compared to those treated with the aid of XSight-spine localization with fiducials (Logrank, $p=0.66)$. Additionally, there was no significant difference in local control or disease-free survival stratified by any tumor tracking method (Logrank, $p=0.44$ and $p=0.97$, respectively) (Fig. 4).

\section{Treatment-related toxicity}

Toxicity was assessed immediately after treatment and again after 3 months of follow-up. Only four patients were documented as developing grade 1 fatigue. No grade 2 or higher toxicity was reported in this cohort at any time (data not shown).

\section{Discussion}

In this study, medically inoperable patients with early or intermediate-stage peripheral lung tumors were treated with SBRT on a CyberKnife treatment platform to a range 


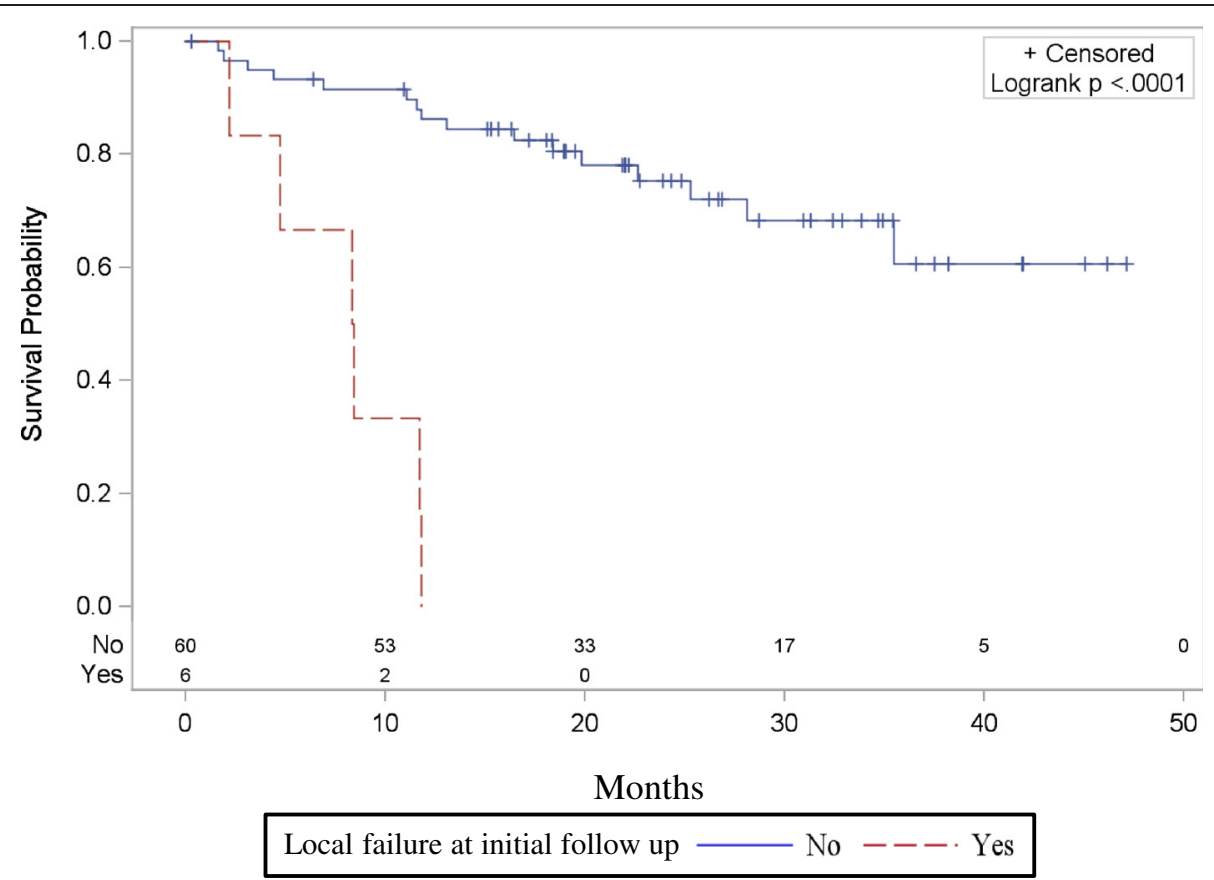

Fig. 1 Achieving initial local control in previously untreated, medically inoperable patients with peripheral tumors targeted with SBRT is a predictor for improved survival. Comparing patients that achieved local control at first follow-up scan after SBRT was significantly associated with overall survival (OS) $(p<0.0001)$. OS at one year was $86.2 \%$ (95\% Cl, 74.3-92.9) in those achieving initial local control, whereas the one year survival probability for patients with local failure at first follow-up scan was $0.0 \%$ (95\% Cl, not estimable)

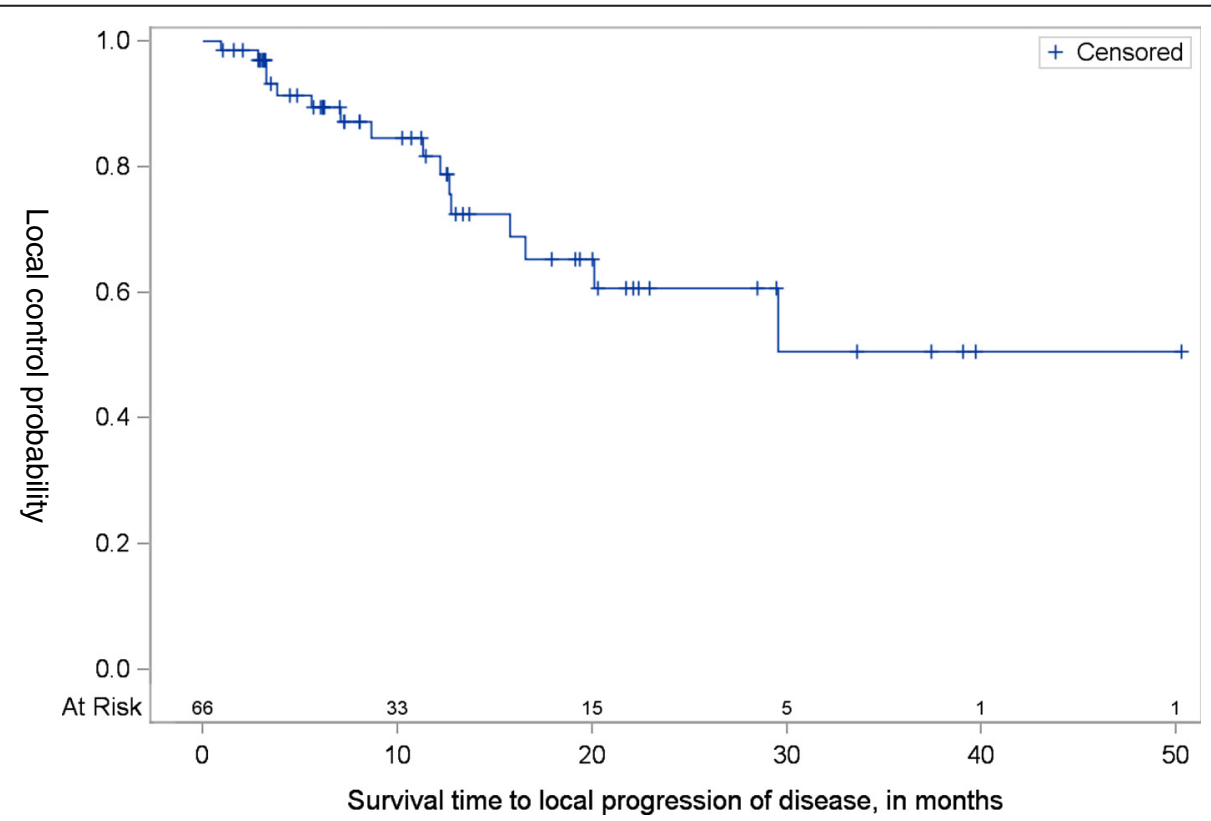

Fig. 2 Local control in previously untreated, medically inoperable patients with peripheral tumors after being treated with Cyberknife SBRT. Local disease control at 12 months was $81.8 \%(95 \% \mathrm{Cl}, 67.3-90.3 \%)$ and at 24 months was $60.6 \%$ (95\% Cl, 41.5- 75.2\%). The median time to local progression of disease was not reached 
A

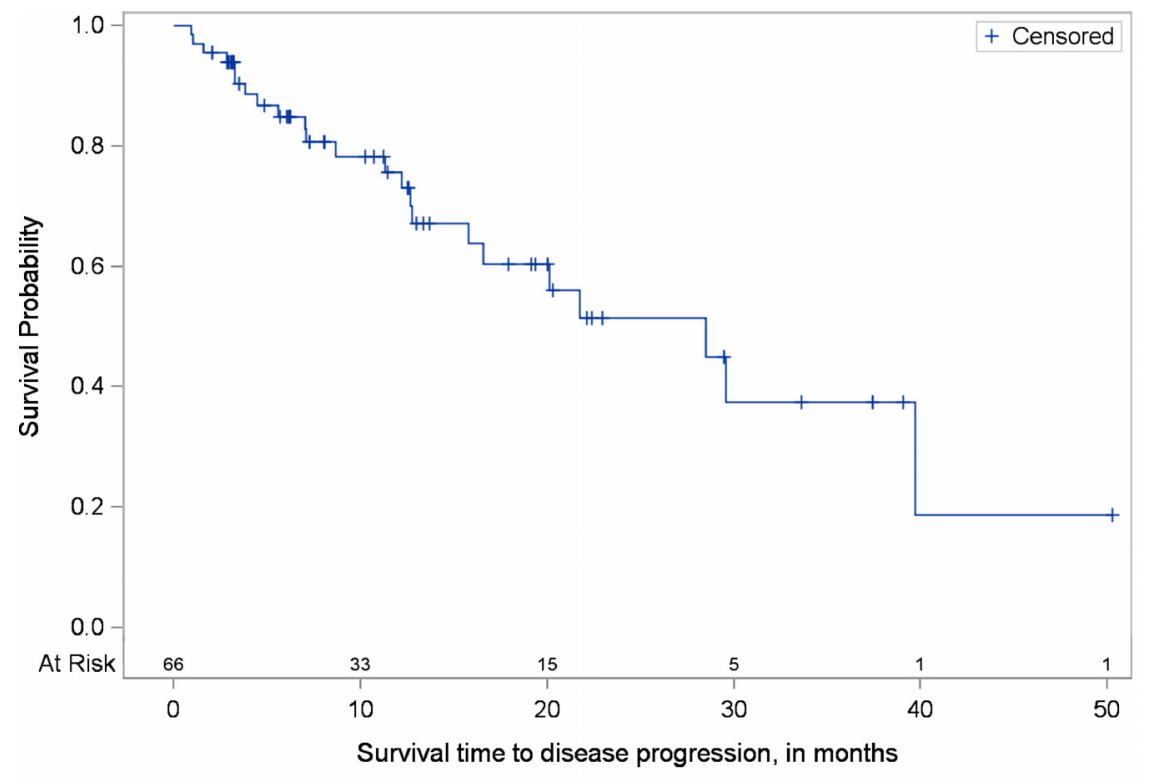

B

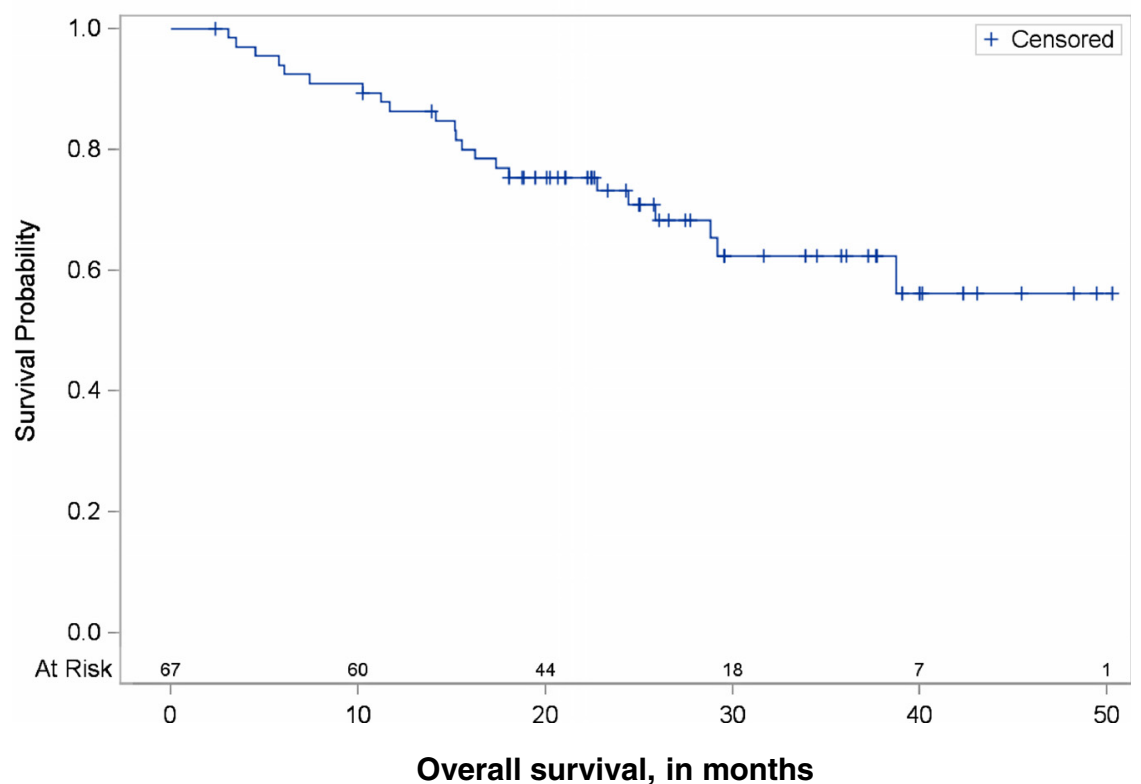

Fig. 3 Disease free and overall survival in previously untreated patients with peripheral tumors deemed to be medically inoperable after being treated with Cyberknife SBRT. a The median progression-free survival was estimated to be 28.5 months ( $95 \%$ Cl: 15.8 months to not reached). The overall disease-free survival at 3 years was $37.5 \%$ (95 \% Cl: 17.7- $57.4 \%$ ). b The overall survival at 3 years (36 months) was $62.4 \%$ (95\% Cl: 74.3-47.3\%). The median overall survival time was not reached

of biological effective doses (BED, 180-85.5 Gy $\mathrm{y}_{10}$ ). The overall survival in our cohort was similar to that achieved in RTOG 0236 (62.4 \% vs. $55.8 \%$ at 36 months) [15]. Toxicity observed in this study, significant only for low grade fatigue, was also improved when compared to earlier studies-one of the first being the Indiana University phase I dose escalation and feasibility trial with 5 treatment-related deaths (pneumonia $(n=3)$, respiratory failure $(n=1)$, and hemoptysis $(n=1)$ occurring throughout the study period out of 70 patients treated with lung SBRT to a total dose of 60-66Gy in three fractions [12]. In light of these findings, we selected more conservative doses in an effort to balance the risk of toxicity with the efficacy of tumor control. In addition, we selected lower doses based on others' observations reporting comparable 3 year local control rates above $80 \%$ at 2 years using BEDs 


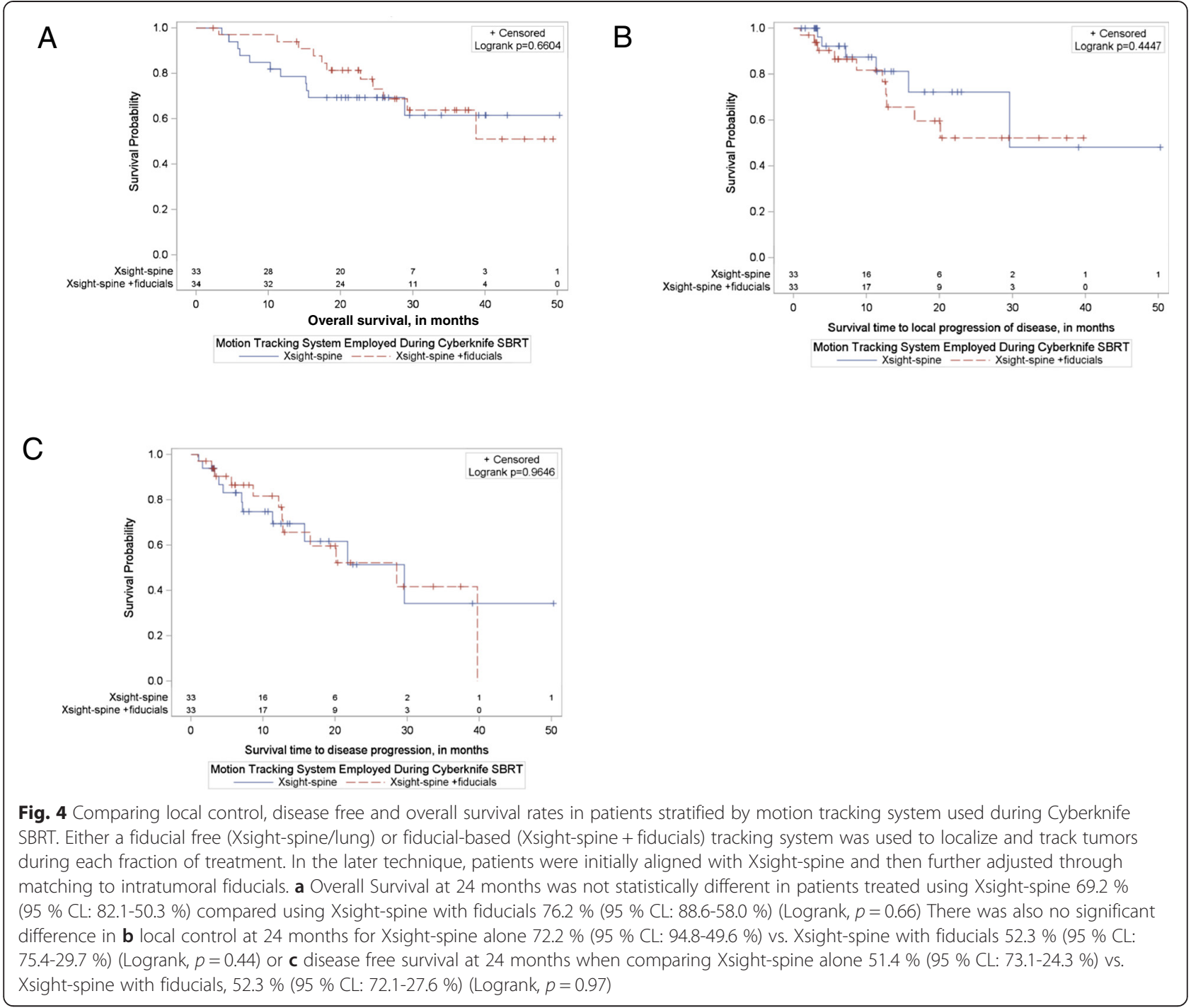

Table 3 Selected studies comparing local control as a function of BED

\begin{tabular}{|c|c|c|c|c|}
\hline Author, year & Stage & Dose, BED10 & Local control & $P$ value \\
\hline \multirow[t]{2}{*}{ Van der Voort van Zyp et al. 2009 [19] } & \multirow[t]{2}{*}{$\mathrm{T} 1, n=35 ; \mathrm{T} 2, n=24$} & $180 \mathrm{~Gy}$ & $96 \%$ at 2 years & \multirow[t]{2}{*}{ n.s. $p=0.19$} \\
\hline & & 112. 5 Gy & $78 \%$ at 2 years & \\
\hline \multirow[t]{2}{*}{ Onimaru et al. 2008 [20] } & \multirow[t]{2}{*}{$\mathrm{T} 1, n=25 ; \mathrm{T} 2, n=16$} & $80 \mathrm{~Gy}$ & 45 at 2 years & \multirow[t]{2}{*}{$p=0.0042$} \\
\hline & & 105.6 Gy & $89 \%$ at 2 years & \\
\hline \multirow[t]{2}{*}{ Guckenberger et al. 2009 [21] } & \multirow[t]{2}{*}{$\mathrm{T} 1, n=13 ; \mathrm{T} 2, n=19 ; \mathrm{T} 3 \mathrm{~N} 0, n=9$} & >100 Gy & $89 \%$ at 3 years & \multirow[t]{2}{*}{$p=0.0001$} \\
\hline & & $<100$ Gy & $62 \%$ at 3 years & \\
\hline \multirow[t]{2}{*}{ Bibault et al. 2012 [6] } & \multirow[t]{2}{*}{$\mathrm{T} 1, n=31 ; \mathrm{T} 2, n=20$} & $\leq 150$ Gy & $70 \%$ at 2 years & \multirow[t]{2}{*}{$p=0.006$} \\
\hline & & >150 Gy & $100 \%$ at 2 years & \\
\hline \multirow[t]{2}{*}{ Grills et al. 2012 [22] } & \multirow[t]{2}{*}{$\mathrm{T} 1, n=318 ; \mathrm{T} 2, n=67 ; \mathrm{T} 3, n=10$} & $<105$ Gy & $85 \%$ at 2 years & \multirow[t]{2}{*}{$p<0.001$} \\
\hline & & >105 Gy & $96 \%$ at 2 years & \\
\hline \multirow[t]{2}{*}{ Onishi et al. 2007 [23] } & \multirow[t]{2}{*}{ Stage $1, n=257$} & $>100 \mathrm{~Gy}$ & $84 \%$ at 3 years & \multirow[t]{2}{*}{$p<0.01$} \\
\hline & & $<100$ Gy & $37 \%$ at 3 years & \\
\hline
\end{tabular}


that approximate the range used in our cohort [17, 18]. In spite of these efforts, local control was lower than expected when compared to these historic controls.

We found that indeed, the local control rate at 24 months had decreased to $60.6 \%$ compared to $91 \%$ at three years seen in RTOG 0236 [15]. Although minor deviations in treatment planning may have contributed to this observation, the lower BEDs employed in this study are thought to be contributing factors compared to those used in earlier studies such as the Indiana University phase I dose escalation trial and RTOG 0236 where BEDs used were considerably higher- approaching 151.2 $\mathrm{Gy}_{10}$ once tissue heterogeneity corrections were applied $[15,12]$. Furthermore, several others have reported that dose escalation correlated directly with significantly improved local control rates (Table 3) [6, 19-23]. It should also be emphasized that $22 \%$ of the patients included in our cohort had stage IB to stage III disease and RTOG 0236 included patients with only T1-T2 tumors. Thus, it is possible that this may contribute to the lower local control rate seen in this study. Nevertheless, caution must be exercised when interpreting these results due to the limitations implicit within a retrospective study such as this. An additional limitation of this study is the relatively small sample size $(n=67$ patients/treated lung tumors) which may explain the lack of statistical significance seen when performing a comparative analysis of survival outcomes to identify predictive factors (Additional file 1: Figure S1 and Additional file 2: Figure S2).

As anticipated, patients that had achieved local control initially at the time of first follow-up had significantly improved overall survival compared with those that did not. These data support the notion that achieving initial local tumor control is of critical importance for survival.

Ablative therapies such as SBRT are now routinely designed without reliance on intra-target fiducial markers since the advent of sophisticated onboard-non-invasive image guidance systems [6, 13, 24]. Several phantom studies have demonstrated the ability to achieve submillimeter targeting with these systems [25]. However, evidence supporting the use of fiducial-free image guidance and localization systems is limited $[6,13,25]$. In this analysis, through a side-by-side comparison, we show that there is no difference statistically in survival outcome or tumor control metrics when stratifying patients by tracking technique used. Thus, non-invasive image-based tracking techniques appear to be safe and effective alternatives compared with traditional fiducialbased targeting and is in agreement with others' reports $[13,18,24,25]$. This has important implications in medically inoperable, frail patient populations with lung cancer that may not be able to easily tolerate such invasive procedures.

\section{Conclusions}

This study supports the role of SBRT in the treatment paradigm for early-stage lung cancer in medically inoperable patients in order to gain local control of their disease, and doing so appears to be critical to achieving prolonged overall survival in this patient population. It provides evidence to justify the use of non-invasive tumor targeting during treatment administration over invasive fiducial-based targeting methods. Lastly, the importance of dose escalation is emphasized by our results especially with regard to the achievement of durable local tumor control. Nevertheless, further studies evaluating higher dose fractionation schemes are warranted to reach a comfortable equilibrium between better local control and low treatment-related morbidity.

\section{Additional files}

Additional file 1: Figure S1. Comparison of disease free survival rates in patients stratified by gender, stage, pathology and location. The different Kaplan-Meier curves for time until disease progression were not found to be statistically significant (using the log-rank test and $p<0.05$ ) among the following subgroups in univariate analysis: (A) Gender at 24 months comparing male patients, $49.3 \%$ (95 \% CL: 71.7-22.4 \%) to female $54.3 \%$ (95 \% CL: 73.6-29.6 \%), ( $p=0.8$ ), (B) Stage at 24 months comparing early stage patients with T1a disease $47.8 \%$ (95 \% CL: 64.9-28.5 \%) to those with intermediate stage IB-III disease, $64.3 \%$ (95 \% CL: 90.2-15.2 \%), $(p=0.16)$, (C) pathology at 24 months for patients with adenocarcinoma $41.4 \%$ (95 \% CL: 66.2-15.2\%), squamous cell carcinoma $30.3 \%$ (95 \% CL: 61.6-5.3\%), and unspecified non-small cell lung $64.3 \%$ (95 \% CL: 90.2-15.2 \%), $(p=0.1)$ or (D) tumor location within the left lower lobe (LL), $50.1 \%(95 \% \mathrm{CL}$. 77.7-15.1 \%), left upper lobe (LU), $73.7 \%$ (95 \% CL: 91.3-35.9\%) right lower lobe (RL), $28.3 \%$ (95 \% CL: 60.0-44.0 \%), or right upper lobe (RU) $46.4 \%$ (95 \% $C L: 75.2-12.6 \%),(p=0.15)$.

Additional file 2: Figure S2. Comparison of overall survival rates in patients stratified by gender, stage, pathology and location. The different Kaplan-Meier curves for overall survival were not found to be statistically significant (using the log-rank test and $p<0.05$ ) among the following subgroups in univariate analysis: (A) gender at 24 months comparing male patients, $87.1 \%$ (95 \% CL: 95.7-65.0 \%) to female patients $65.9 \%$ (95\% CL: 78.3-49.2\%) ( $p=0.8)$, (B) stage at 24 months comparing early stage patients with T1a disease $69.7 \%$ (95 \% CL: 80.5-55.7 \%) to those with intermediate stage IB-III disease, $86.7 \%$ (95 \% CL: 96.5-56.4 \%) $(p=0.37)$, (C) pathology at 24 months for patients with adenocarcinoma $75.4 \%$ (95 \% CL: 87.5-55.2 \%), squamous cell carcinoma $71.4 \%$ (95 \% CL: 88.2-40.6\%), and unspecified non-small cell lung $70.7 \%$ (95 \% CL: 87.9-39.4 \%) ( $p=0.77)$, or (D) tumor location within the left lower lobe (LL), 68.8 \% (95 \% CL: 85.6-40.5 \%), left upper lobe (LU), 68.2 \% (95 \% CL: 85.4-39.5\%), right lower lobe (RL), $90.0 \%$ (95\% CL: 98.5-47.3\%), or right upper lobe (RU), $73.9 \%$ (95\% CL: 87.3-50.9\%) $(p=0.81)$.

\section{Competing interests}

The authors declare that they have no competing interests.

\section{Authors' contributions}

All authors contributed to the design, data collection, analysis and writing of this work. All authors read and approved the final manuscript.

\section{Acknowledgments}

We would like to acknowledge and thank Dr. David Collingridge for his valued advice and assistance in editing and critiquing this manuscript. 


\section{Submission}

This material has never been published and is not currently under evaluation in any other peer-reviewed publication. All authors have participated in the clinical care, data acquisition and review, and preparation of this manuscript. This work was presented at the September, 2014 American Society of Radiation Oncology annual meeting in San Francisco, CA.

\section{Author details}

${ }^{1}$ The Department of Radiation Medicine, North Shore-LIJ Cancer Institute, Lake Success, NY, USA. ${ }^{2}$ The Department of Radiation Medicine, North Shore-LIJ Health System, Huntington, NY, USA. ${ }^{3}$ The Department of Biostatistics, Feinstein Institute for Medical Research, North Shore-LIJ Health System, Manhasset, NY, USA. ${ }^{4}$ 989W Jericho Turnpike, Smithtown, NY 11787, USA.

Received: 26 January 2015 Accepted: 18 May 2015

Published online: 28 May 2015

\section{References}

1. Timmerman RD, Herman J, Cho LC. Emergence of stereotactic body radiation therapy and its impact on current and future clinical practice. J Clin Oncol. 2014:32:2847-54.

2. Rowell NP, Williams CJ. Radical radiotherapy for stage $1 / /$ non-small cell lung cancer in patients not sufficiently fit for or declining surgery (medically inoperable): a systematic review. Thorax. 2001;56:628-38.

3. Potters L, Steinberg M, Rose C, Timmerman R, Ryu S, Hevezi JM, et al. American Society for Therapeutic Radiology and Oncology and American College of Radiology practice guideline for the performance of stereotactic body radiation therapy. Int J Radiat Oncol Biol Phys. 2004;60:1026-32.

4. Potters L, Kavanagh B, Galvin JM, Hevezi JM, Janjan N, Larson D, et al. American Society for Therapeutic Radiology and Oncology (ASTRO) and American College of Radiology (ACR) practice guideline for the performance of stereotactic body radiation therapy. Int J Radiat Oncol Biol Phys. 2010;76:326-32.

5. Ryu S, Yoon H, Stessin A, Gutman F, Rosiello A, Davis R. Contemporary treatment with radiosurgery for spine metastasis and spinal cord compression in 2015. Radiat Oncol J. 2015;33:1-11.

6. Bibault J-E, Prevost B, Dansin E, Mirabel X, Lacornerie T, Lartigau E. Imageguided robotic stereotactic radiation therapy with fiducial-free tumor tracking for lung cancer. Radiat Oncol. 2012;7:102.

7. Dosoretz D, Katin M, Blitzer P, Rubenstein J, Galmarini D, Garton G, et al. Medically inoperable lung carcinoma: the role of radiation therapy. Semin Radiat Oncol. 1996;6:98-104.

8. Stieb S, Lang S, Linsenmeier C, Graydon S, Riesterer O. Safety of high-doserate stereotactic body radiotherapy. Radiat Oncol. 2015;10:4-11.

9. Amini A, Yeh N, Gaspar LE, Kavanagh B, Karam SD. Stereotactic Body Radiation Therapy ( SBRT) for lung cancer patients previously treated with conventional radiotherapy: a review. Radiat Oncol. 2014;9:2-9.

10. Karam SD, Horne ZD, Hong RL, McRae D, Duhamel D, Nasr NM. Dose escalation with stereotactic body radiation therapy boost for locally advanced non small cell lung cancer. Radiat Oncol. 2013;8:179.

11. Yamashita H, Haga A, Takahashi W, Takenaka R, Imae T, Takenaka S. Volumetric modulated arc therapy for lung stereotactic radiation therapy can achieve high local control rates. Radiat Oncol. 2014;9:1-6.

12. Fakiris AJ, McGarry RC, Yiannoutsos CT, Papiez L, Williams M, Henderson MA, et al. Stereotactic body radiation therapy for early-stage non-small-cell lung carcinoma: four-year results of a prospective phase II study. Int J Radiat Oncol Biol Phys. 2009;75:677-82.

13. Bahig H, Campeau M-P, Vu T, Doucet R, Béliveau Nadeau D, Fortin B, et al. Predictive parameters of CyberKnife fiducial-less (XSight Lung) applicability for treatment of early non-small cell lung cancer: a single-center experience. Int J Radiat Oncol Biol Phys. 2013;87:583-9.

14. Hertz-Picciotto I, Rockhill B. Validity and efficiency of approximation methods for tied survival times in Cox regression. Biometrics. 1997;53:1151-6.

15. Timmerman R, Paulus R, Galvin J, Michalski J, Straube W, Bradley J, et al. Stereotactic body radiation therapy for inoperable early stage lung cancer. JAMA. 2010;303:1070-6.

16. Edge SB, Byrd DR, Compton CC, Fritz AG, Greene FL, Trotti A. AJCC Cancer Staging Manual. 7th ed. New York: Springer; 2010. p. 253-70.
17. Brown WT, Wu X, Fayad F, Fowler JF, Amendola BE, García S, et al. Cyberknife radiosurgery for stage I lung cancer: results at 36 months. Clin Lung Cancer. 2007:8:488-92.

18. Swangsilpa T, Yongvithisatid P, Pairat K, Dechsupa P, Dhanachai M, Dangprasert S, et al. Preliminary experience of CyberKnife treatment of primary non-small cell lung cancer. J Med Assoc Thai. 2012;95:1335-43.

19. Van der Voort van Zyp NC, Prévost J-B, Hoogeman MS, Praag J, van der Holt B, Levendag PC, et al. Stereotactic radiotherapy with real-time tumor tracking for non-small cell lung cancer: clinical outcome. Radiother Oncol. 2009;91:296-300.

20. Onimaru R, Fujino M, Yamazaki K, Onodera Y, Taguchi H, Katoh N, et al. Steep dose-response relationship for stage I non-small-cell lung cancer using hypofractionated high-dose irradiation by real-time tumor-tracking radiotherapy. Int J Radiat Oncol Biol Phys. 2008;70:374-81.

21. Guckenberger M, Wulf J, Mueller G, Krieger T, Baier K, Gabor M, et al. Dose response relationship for image-guided stereotactic body radiotherapy of pulmonary tumors: relevance of $4 \mathrm{D}$ dose calculation. Int J Radiat Oncol. 2009; $74: 47-54$

22. Grills IS, Hope AJ, Guckenberger M, Kestin LL, Werner-Wasik M, Yan D, et al. A collaborative analysis of stereotactic lung radiotherapy outcomes for early-stage non-small-cell lung cancer using daily online cone-beam computed tomography image-guided radiotherapy. J Thorac Oncol. 2012;1382-1393.

23. Onishi H, Shirato H, Nagata Y, Hiraoka M, Fujino M, Gomi K, et al. Hypofractionated stereotactic radiotherapy (HypoFXSRT) for stage I non-small cell lung cancer: updated results of 257 patients in a Japanese multi-institutional study. J Thorac Oncol. 2007;2:94-100.

24. Bahig H, Filion E, Vu T, Roberge D, Lambert L, Bouchard M, et al. Excellent Cancer Outcomes Following Patient-adapted Robotic Lung SBRT But a Case for Caution in Idiopathic Pulmonary Fibrosis. Technol Cancer Res Treat. 2014. Epub ahead of print.

25. Bibault JE, Prevost B, Dansin E, Mirabel X, Lacornerie T, Dubus F, et al. Stereotactic radiotherapy for lung cancer: non-invasive real-time tumor tracking. Cancer Radiother. 2010;14:690-7.

\section{Submit your next manuscript to BioMed Central and take full advantage of:}

- Convenient online submission

- Thorough peer review

- No space constraints or color figure charges

- Immediate publication on acceptance

- Inclusion in PubMed, CAS, Scopus and Google Scholar

- Research which is freely available for redistribution 